\title{
Đánh giá hiệu suất mạng LTE sử dụng kỹ thuật TDD linh động
}

\author{
Lâm Sinh Công \\ Trường Đại học Công nghệ, Đại học Quốc Gia Hà Nội \\ Liên hệ: congls@vnu.edu.vn \\ Ngày nhận bài: 19/07/2019, ngày sửa chữa: 04/09/2019, ngày duyệt đăng: 06/09/2019 \\ Xem sớm trực tuyến: 09/09/2019, định danh DOI: 10.32913/mic-ict-research-vn.v2019.n1.876 \\ Biên tập lĩnh vực điều phối phản biện và quyết định nhận đăng: TS. Trương Trung Kiên
}

Tóm tắt: Kỹ thuật tái sử dụng tần số theo tỉ lệ được coi là một trong những kỹ thuật then chốt để tăng dung lượng mạng của hệ thống mạng LTE (4G, 5G). Bên cạnh đó, kỹ thuật song công phân chia thời gian thích nghi (TDD thích nghi) cũng được coi là một kỹ thuật tiên tiến trong hệ thông tin di động thế hệ mới 5G. Tuy nhiên, việc đánh giá hiệu suất hệ thống mạng LTE sử dụng đồng thời hai kỹ thuật này chưa được nghiên cứu một cách đầy đủ. Trong bài báo này, chúng ta sử dụng mô hình xác suất thống kê để mô hình hóa hai kỹ thuật này trong mạng LTE. Tham số dùng để đánh giá trong bài báo là xác suất phủ sóng của người dùng biên (cách xa trạm phục vụ). Bài báo đưa ra biểu thức xác suất phủ sóng của người dùng đồng biên thời phân tích ảnh hưởng của các tham số liên quan đến xác suất này. Bài báo đưa ra một kêt luận quan trọng cho việc thiết kế mạng: để tăng xác suất phủ sóng của người dùng biên, việc giảm số lượng trạm cơ sở sử dụng cùng một sóng mang tại cùng một thời điểm mang lại hiệu quả cao hơn việc tăng số lượng trạm phát trong mạng.

Từ khóa: Xác suất phủ sóng, TDD linh động, kỹ thuật tái sử dụng tần số, mạng Poisson, LTE.

Title: Performance Analysis of LTE Networks Enabling Dynamic TDD

Abstract: Fractional Frequency Reuse is one of the most popular techniques to improve the performance of LTE networks (4G and beyond). Recently, Dynamic Time Division Duplex (dynamic TDD) has been introduced as the potential technique for $5 \mathrm{G}$ cellular networks. However, the co-existence of these techniques in $5 \mathrm{G}$ networks has not been fully investigated. In this paper, we ultilize stochastic geometry to model and evaluate the performance of cellular networks which deploy both fractional frequency reuse and dynamic TDD. The paper derives the average coverage probability of the cell edge user and then analyze the effects of the network parameters on this coverage probability. An interesting fact is found in this paper which states that reducing the number of interfering base stations is more important than increasing the number of base stations in the networks.

Keywords: Coverage probability, dynamic TDD, fractional frequency reuse, Poisson point networks, LTE networks.

\section{GIỚI THIÊU}

Sự bùng nổ về số lượng thuê bao cũng như lưu lượng dữ liệu trong mạng những năm gần đây đã thúc đẩy các nhà mạng và các tổ chức nghiên cứu và phát triển hệ thống mạng thế hệ thứ 5 (5G) LTE [1]. Theo các hệ tiêu chuẩn được công bố tại phiên bản (Release) số 15 vào năm 2018 [2] và các phiên bản sau này, hệ thống thông tin di động $5 \mathrm{G}$ sẽ kế thừa một số kỹ thuật tiêu biểu của 4G LTE (LongTerm Evolution) [3] như kỹ thuật tái sử dụng tần số theo tỉ lệ, hay gọi tắt là kỹ thuật tái sử dụng tần số, và tích hợp một số kỹ thuật mới như kỹ thuật song công phân chia theo thời gian linh động (Dynamic TDD: Dynamic Time Division Duplexing).

Kỹ thuật tái sử dụng tần số $[4,5]$ là một kỹ thuật truyền thống của mạng thông tin di động cho phép các trạm khác nhau sử dụng chung một dải tần số. Tuy nhiên đối với các hệ thống trước đây, các trạm muốn sử dụng tần số phải ở những vị trí cách rất xa nhau để tránh gây nhiễu truyền tin giữa các trạm. Đối với hệ thống LTE, kỹ thuật tái sử dụng tần số được cải tiến để cho phép những trạm cạnh nhau cũng có thể tái sử dụng tần số của nhau. Trong hệ thống 5G mật độ các trạm phát trở lên dày đặc và có thể lớn hơn 100 trạm $/ \mathrm{km}^{2}$ [6], kỹ thuật tái sử dụng tần số được áp dụng như một kỹ thuật then chốt để phân chia nguồn tài nguyên cho các thiết bị.

Kỹ thuật TDD linh động cải tiến từ kỹ thuật TDD được giới thiệu cho hệ thống mạng 4G LTE thông qua các phiên bản từ 8 đến 14. Trong đó, mỗi trạm di động sử dụng cùng một sóng mang để trao đổi thông tin với trạm cơ sở nó kết nối. Khi đó việc truyền và nhận tin diễn ra trên sóng mang đó một cách luôn phiên. Như vậy, với kỹ thuật TDD, mỗi 


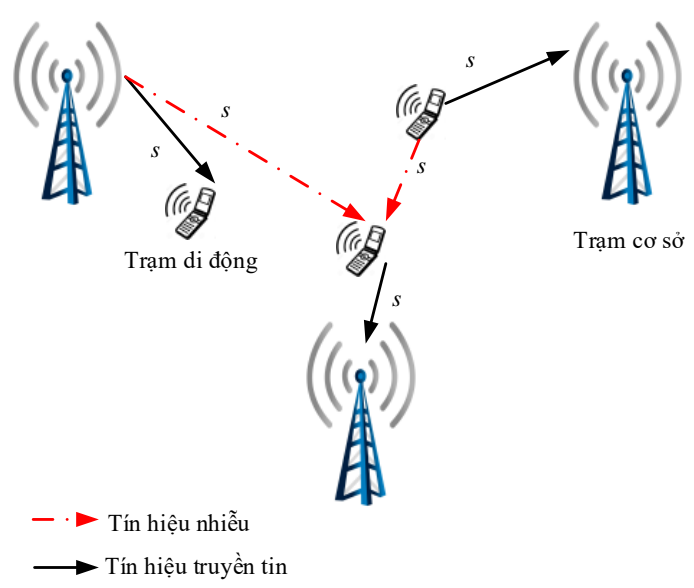

Hình 1. Chia sẻ sóng mang giữa trạm di động và trạm cơ sở.

sóng mang được chia sẻ giữa trạm cơ sở và người dùng. Điều đó có nghĩa là một sóng mang được sử dụng cho đường xuống bởi một trạm phát nào đó có thể được tái sử dụng cho đường lên bởi trạm phát lân cận. Như vậy, một người dùng bất kỳ trong mạng có thể chịu ảnh hưởng bởi nhiễu từ cả trạm cơ sở và trạm di động.

Các phiên bản trên cũng đã giới thiệu kỹ thuật TDD bán linh động (semi-dynamic) để lựa chọn cấu hình về tỉ lệ về số khe thời gian giữa đường lên và đường xuống. Tuy nhiên tỉ lệ về số khe thời gian này thường là cố định và do đó kỹ thuật này còn bộc lộ nhiều hạn chế khi lượng tải trên đường lên hoặc đường xuống biến đổi nhanh. Do đó, kỹ thuật TDD linh động $[7,8]$ đã được giới thiệu và thu hút rất nhiều sự quan tâm. Với kỹ thuật này, việc cấp phát khe thời gian được thay đổi một cách linh hoạt theo lưu lượng tải trên cả đường lên và đường xuống, đề từ đó tăng được dung lượng hệ thống $[9,10]$. Hình 1 là một ví dụ về kỹ thuật TDD.

Hiện tại, đã có rất nhiều bài báo khoa học nghiên cứu tính khả thi của kỹ thuật TDD linh động trong mạng 5G. Các tác giả trong tài liệu [11] dựa trên mô hình xác suất thống kê để đánh giá hiệu suất của kỹ thuật TDD linh động. Tuy nhiên, các biểu thức đưa ra chưa chỉ ra rõ ràng ảnh hưởng của kỹ thuật này tới hiệu suất hệ thống. Các kết quả phân tích cụ thể đạt được dựa trên mô hình mô phỏng. Tài liệu [12] đã phân tích tính khả thi của việc áp dụng kỹ thuật TDD linh động đối với hệ thống LTE thế hệ mới, cụ thể là mạng thông tin di động $5 \mathrm{G}$. Trong tài liệu $[7,8]$, hiệu suất của kỹ thuật TDD linh động đã được phân tích trong hệ thống mạng siêu dày đặc, một trong những mô hình mạng của hệ thông tin di động 5G. Ngoài ra, tài liệu [10] còn so sánh hiệu suất của kỹ thuật TDD linh động và TDD bán linh động trong điều kiện truyền tin có xuất hiện đường truyền thẳng và không xuất hiện đường truyền thẳng. Các kết quả trên đều cho thây kỹ thuật TDD linh động hoàn toàn có thể mang lại những lợi ích như tối ưu hóa hiệu suất phổ, nâng cao hiệu suất người dùng.

Các tài liệu trên đã đưa ra rất nhiều phương pháp có thể phân tích hệ suất của kỹ thuật TDD linh động. Tuy nhiên, các tài liệu trên chưa đề cập chi tiết đến kỹ thuật tái sử dụng tần số. Trong các mô hình phân tích, các tác giả đều giả thiết rằng kỹ thuật tái sử dụng tần số toàn phần (hệ số tái sử dụng $\Delta=1$ ) được sử dụng. Với hệ số tái sử dụng tần số $\Delta=1$, tất cả người dùng được phục vụ cùng với một mức công suất. Tuy nhiên, phương pháp này gây ra nhiễu nghiêm trọng cho người dùng và gây ra sự suy hao mạnh về hiệu suất hệ thống [5]. Đặc biệt phương pháp này không đáp ứng được mục tiêu của các kỹ thuật tái sử dụng tần số theo theo khuyến nghị của 3GPP là làm tăng hiệu suất của những người dùng có kênh truyền kém hoặc bị suy hao mạnh bởi môi trường.

Trong bài báo này, chúng ta mô hình hóa mạng di động sử dụng đồng thời kỹ thuật TDD linh động và kỹ thuật tái sử dụng tần số với hệ số tái sử dụng $\Delta>1$. Hiệu suất của mạng được phân tích thông qua xác suất phủ sóng của người dùng ở biên, là những người dùng có tỉ số tín hiệu trên nhiễu cộng ồn (SINR: Signal-to-Interference-plusNoise) thấp. Bài báo đưa ra được biểu thức tính xác suất phủ sóng của người dùng đồng thời phân tích ảnh hưởng của các tham số như ngưỡng phủ sóng, mật độ trạm phát và tỉ lệ trạm cơ sở/trạm di động sử dụng cùng sóng mang lên hiệu suất của mạng.

\section{MÔ HİNH MANG}

Trong bài báo này, chúng ta mô hình các trạm phát theo các thông số sau:

- Mật độ trạm phát là một biến ngẫu nhiên tuân theo tiến trình Poisson không gian (Spatial PPP: Spatial Point Poisson Process), như hình 2, có tham số giá trị trung bình là $\lambda$;

- Vị trí các trạm được phân bố một các ngẫu nhiên theo phân bố chuẩn;

- Người dùng được giả thiết kết nối với trạm gần nhất ở khoảng cách $r$. Hàm mật đô (PDF: Probability Density Function) của khoảng cách giữa người dùng và trạm nó kết nối là

$$
f_{R}(r)=2 \pi \lambda r e^{-\pi \lambda r^{2}}
$$

Trọng tâm của bài báo là phân tích hiệu suất của người dùng được phục vụ bởi sóng mang con (sub-carrier) $s$. Ta giả thiết rằng tất cả các trạm lân cận được cấp phát sóng mang con $s$ đều đang phát trên sóng mang con này. Trên thực tế, nếu như một trạm phát ni không phát trên sóng mang con $s$ thì sự xuất hiện của trạm $n i$ này không ảnh 


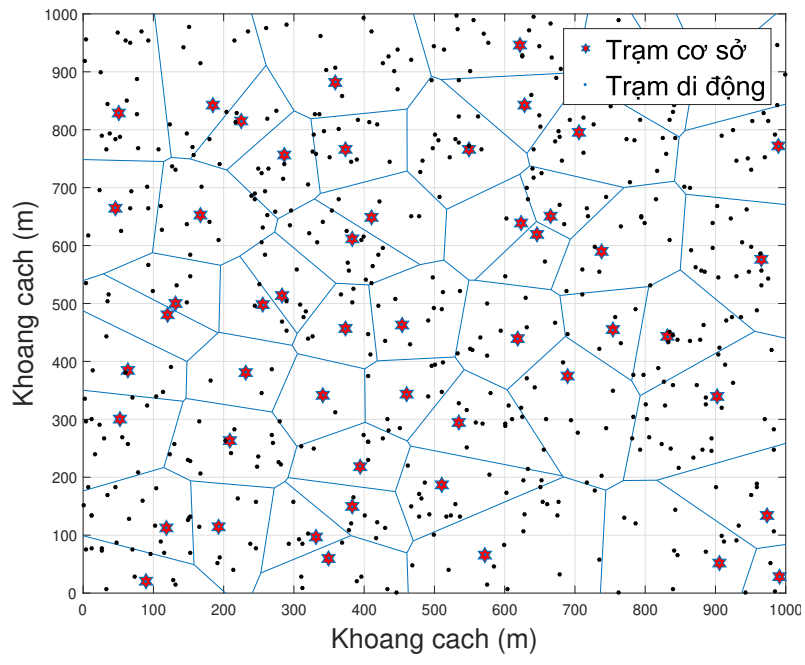

Hình 2. Ví dụ về mô hình mạng PPP.

hưởng đến hiệu suất của người dùng trên sóng mang này. Khi đó, trạm phát ni không thuộc phạm vi xem xét của bài báo và mật độ trạm phát vẫn tuân theo phân bố Poisson nhưng với tham số giá trị trung bình là $\rho \lambda(0<\rho<1)$.

Ta giả thiết rằng phương pháp truyền $\mathrm{TDD}$ linh động kết hợp với phương pháp tái sử dụng tần số cứng được sử dụng.

\section{Kỹ thuật tái sử dụng tần số}

Ý tưởng của kỹ thuật tái sử dụng tần số cứng là chia người dùng và số sóng mang được cấp thành các nhóm với mục đích là mỗi nhóm người dùng được phục vụ bởi một nhóm sóng mang như Hình 3 . Thông thường người dùng sẽ được phân chia thành người dùng trung tâm (CCU: CellCenter User) và người dùng biên (CEU: Cell-Edge User). Trong đó, những người dùng được có tỉ số SINR của nó lớn hơn ngưỡng SINR định trước thì được phân loại thành CCU, và những người dùng còn lại được phân loại thành CEU. Do các CCU chia sẻ toàn bộ nguồn tài nguyên với nhau nên mật độ trạm phát sử dụng sóng mang $s$ để phục vụ CCU là $\lambda^{(c)}=\lambda$. Ngược lại, do các CEU trong một nhóm $\Delta$ trạm không chia sẻ các sóng mang với nhau nên mật độ các trạm sử dụng sóng mạng $s$ để phục vụ CEU là $\lambda^{(e)}=\lambda / \Delta$, trong đó $\Delta$ được gọi là hệ số tái sử dụng tần số.

Tiếp theo chúng ta sẽ tập trung xây dựng biểu thức về SINR của người dùng trong pha thiết lập và pha truyền tin. Đối với pha thiết lập, tương tự như giả thiết trong các nghiên cứu trước đây cũng như khuyến nghị của $3 \mathrm{GPP}$, tỉ số SINR trên kênh điều khiên sẽ được sử dụng để phân loại người dùng thành $\mathrm{CCU}$ hoặc $\mathrm{CEU}$. Do các trạm phát trong cùng một hệ thống mạng sử dụng cùng sóng mang và cùng một mức công suất để truyền các thông tin điều

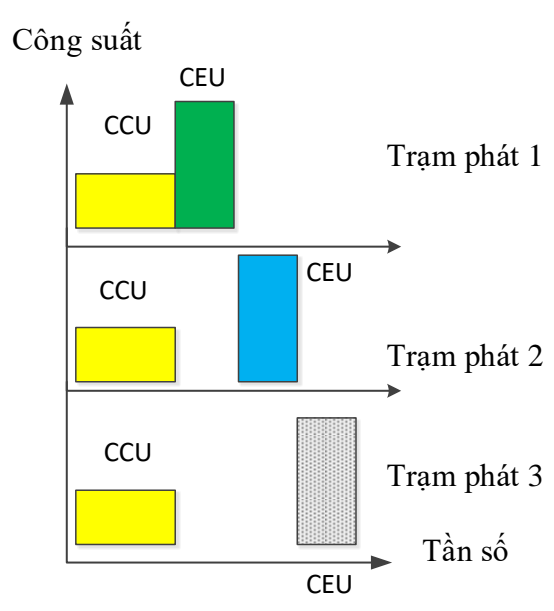

Hình 3. Mô hình kỹ thuật tái sử dụng tần số cứng.

khiển, mỗi kênh điều khiển sẽ chịu ảnh hưởng nhiễu từ tất cả các trạm lân cận.

Công suất nhiễu thu được trên kênh điều khiển đường xuống của người dùng CEU $u$ là

$$
I^{(o)}=\sum_{j \in \theta} P_{d} g_{j} l_{j}^{-\alpha},
$$

trong đó $P_{d}$ là công suất phát trên kênh điều khiển đường xuống, $g_{j}$ và $r_{j}$ là độ lợi công suất của kênh truyền và khoảng cách từ trạm nhiễu thứ $j$ đến người dùng $u, \alpha$ là hệ số suy hao của môi trường truyền tin, $\theta$ là tập hợp chứa tất cả các trạm phát cơ sở gây nhiễu lên kênh điều khiển.

Khi đó tỉ số SINR trên kênh điều khiển là

$$
\operatorname{SINR}^{(o)}=\frac{P_{d} g l^{-\alpha}}{I^{(o)}+\sigma^{2}},
$$

trong đó $l$ là khoảng cách từ CEU $u$ đến trạm nó kết nối và $\sigma^{2}$ là công suất của ồn Gauss.

Đối với pha truyền tin, do CEU thường được phục vụ với công suất cao hơn công suất phát trên kênh điều khiển, ta đặt công suất phát của $\mathrm{CEU}$ là $\phi P_{d}$, với $(\phi>1)$. Quá trình truyền tin được thực hiện trên kênh dữ liệu và sử dụng kỹ thuật TDD linh động. Công suất tín hiệu nhận SINR trong giai đoạn này sẽ được phân tích trong phần tiếp theo.

\section{Song công phân chia thời gian linh động}

Theo phương pháp TDD linh động, một sóng mang có thể được sử dụng cho đường xuống và được tái sử dụng cho đường lên ở trạm lân cận. Như vậy trong trường hợp mạng đạt tải cao nhất thì tất cả các trạm phát được cấp phép sử dụng sóng mang con $s$ (bao gồm cả trạm cơ sở và thiết bị di động) đều phát trên sóng mang này. Khi đó số lượng trạm nhiễu tới CEU sẽ chính là số lượng trạm phát trong mạng trừ đi 1 (trừ đi trạm đang phục vụ CEU đó). 
Ta đặt $\epsilon$ là tỉ lệ số trạm phát trên sóng mang con $s$ là trạm cơ sở, tỉ lệ số trạm phát trên sóng mang con này là trạm di động sẽ là $1-\epsilon$. Đặt $\theta_{d}^{(s)}$ và $\theta_{u}^{(s)}$ lần lượt là tập hợp các trạm nhiễu cơ sở và trạm nhiễu di đông đối với CEU $u$ trên sóng mang $s$ tại pha truyền tin. Khi đó mật độ các trạm phát trong $\theta_{d}^{(s)}$ và $\theta_{u}^{(s)}$ tương ứng là $\epsilon \lambda^{(s)}$ và $(1-\epsilon) \lambda^{(s)}$.

Lưu ý $\theta$ là tập hợp chứa tất cả các trạm phát cơ sở gây nhiễu lên kênh điều khiển của người dùng CEU $u$ tại pha thiết lập nên $\theta_{d}^{(s)}$ sẽ là tập con của $\theta$. Tuy nhiên, $\theta_{u}^{(s)}$ và $\theta$ là hai tập hoàn toàn độc lập nhau.

Đặt $l$ và $d$ là khoảng cách từ CEU $u$ tới trạm nhiễu cơ sở và trạm nhiễu di động. Ta lưu ý rằng do CEU $u$ kết nối với trạm cơ sở gần nhất ở khoảng cách $r$ nên ta có $r<d$. Tuy nhiên, trạm nhiê̂u di động thì có thể ở ngay cạnh CEU $u$, do đó gần như không có sự tương quan giữa $d$ và $r$.

Tổng công suất nhiễu tại CEU $u$ được tính theo công thức sau:

$$
I^{(e)}=\sum_{j \in \theta_{d}^{(e)}} P_{d}^{(e)} g_{j} l_{j}^{-\alpha}+\sum_{j \in \theta_{u}^{(e)}} P_{u}^{(e)} g_{u} d_{j}^{-\alpha},
$$

trong đó $P_{d}^{(e)}$ và $P_{u}^{(e)}$ lần lượt là công suất phát của trạm nhiễu cơ sở và trạm nhiễu di động trên sóng mang $s, g_{d}$ và $g_{u}$ là độ lợi công suất của kênh, $\alpha$ là hệ số suy hao của môi trường truyền sóng.

- Các giá trị công suất được xác định như sau:

$$
\left\{\begin{array}{l}
P_{d}^{(e)}=\phi P_{d} \\
P_{u}^{(e)}=\phi P_{u}
\end{array}\right.
$$

Ta đặt $\eta=P_{u}^{(e)} / P_{d}^{(e)}$. Tỉ số SINR thu được tại người dùng $\mathrm{CEU} u$ trong pha truyền tin là

$$
\operatorname{SINR}^{(s)}=\frac{P_{d}^{(e)} g^{\prime} r^{-\alpha}}{I^{(s)}+\sigma^{2}}
$$

trong đó $g^{\prime}$ là độ lợi công suất của kênh truyền tại pha truyền tin.

Trong bài báo này, chúng ta giả thiết độ lợi của kênh truyền tuân theo hàm phân bố Rayleigh. Do đó, độ lợi công suất sẽ của kênh truyền sẽ tuân theo phân bố mũ với hàm phân bố mật độ và tích lũy lần lượt là

$$
\begin{aligned}
& f_{G}(g)=\exp (-g), \\
& F_{G}(g)=1-\exp (-g) .
\end{aligned}
$$

Hàm sinh momen (MGF: Moment Generating Function) của biến $G$ được tính theo biểu thức sau:

$$
M(g)=E[\exp (-s g)]=\frac{1}{1+s g} .
$$

\section{HIỆU SUẤT HỆ THỐNG}

Đối với mô hình mạng tuân theo phân bố Poisson không gian, thông số phổ biến được sử dụng để đánh giá hiệu suất hệ thống chính là xác suất người dùng được nằm trong vùng phủ sóng của mạng, hay gọi tắt là xác suất phủ sóng. Trong các tài liệu trước đây, chúng ta đã định nghĩa xác suất phủ sóng cho CEU cụ thể như sau.

Sự kiện phủ người dùng được phân loại thành $\mathrm{CEU}$ là $\left(\operatorname{SINR}^{(o)}<T\right)$, trong đó $T$ là ngưỡng SINR dùng để phân loại người dùng thành $\mathrm{CCU}$ hoặc $\mathrm{CEU}$. Sự kiện $\mathrm{CEU}$ nằm trong vùng phủ sóng của mạng là $(\operatorname{SINR}>\hat{T})$, trong đó $\hat{T}$ là giá trị SINR tối thiểu được yêu cầu để truyền tin giữa CEU và trạm cơ sở.

Do đó xác suất phủ sóng của $\mathrm{CEU}$ được định xác định bằng biểu thức xác suất có điều kiện sau:

$$
\mathcal{P}(T, \hat{T}, \epsilon)=\mathbb{P}\left(\operatorname{SINR}>\hat{T} \mid \operatorname{SINR}^{(o)}<T\right) .
$$

Ta lưu ý:

- SINR và $\operatorname{SINR}^{(o)}$ đều là các giá trị ngẫu nhiên và phụ thuộc vào các biến ngẫu nhiên như khoảng cách giữa các trạm, độ lợi công suất của kênh truyền. Do đó, xác suất phủ sóng được tính thông qua việc tính kỳ vọng của xác suất có điều kiện với các biến ngẫu nhiên trên.

- Ta sử dụng ký hiệu $\mathbb{P}$ thay cho ký hiệu $P$ khi định nghĩa biểu thức xác suất để biểu hiện xác suất ở đây là xác suất có điều kiện và được tính qua việc lấy giá trị kỳ vọng.

Đối với hệ thống mạng thực tế, giá trị $T$ có thể được thiết lập bởi nhà mạng để đạt được tỉ lệ CCU và CEU nhất đinh còn giá trị $\hat{T}$ phụ thuộc vào khả năng thu phát của thiếu bị người dùng.

Áp dụng định lý Bayes, ta có

$$
\begin{aligned}
\mathcal{P}(T, \hat{T}, \epsilon) \\
=\frac{\mathbb{P}\left(\operatorname{SINR}>\hat{T}, \mathrm{SINR}^{(o)}<T\right)}{\mathbb{P}\left(\operatorname{SINR}{ }^{(o)}<T\right)} \\
=\frac{\int_{0}^{\infty} \mathbb{P}\left(\operatorname{SINR}>\hat{T}, \operatorname{SINR}^{(o)}<T \mid r\right) f_{R}(r) d r}{\int_{0}^{\infty} \mathbb{P}\left(\operatorname{SINR}^{(o)}<T \mid r\right) f_{R}(r) d r},
\end{aligned}
$$

trong đó $f_{R}(r)$ là hàm phân bố mật độ xác suất của biến $r$ được định nghĩa ở phương trình (1).

\section{Tính $P\left(\operatorname{SINR}>\hat{T}, \operatorname{SINR}^{(o)}<T \mid r\right)$}

Phương pháp tính giá trị $P\left(\operatorname{SINR}>\hat{T}, \operatorname{SINR}^{(o)}>T \mid r\right)$ đã được trình bày trong các tài liệu gần đây. Trong bày này, 
chúng ta chỉ nhấn mạnh lại các bước cơ bản để tính toán và sự khác biệt trong kết quả. Ta có

$$
\begin{aligned}
& P\left(\operatorname{SINR}>\hat{T}, \operatorname{SINR}^{(o)}<T \mid r\right) \\
& =P\left(\frac{P_{d}^{(e)} g^{\prime} r^{-\alpha}}{I^{(e)}+\sigma^{2}}>\hat{T}, \frac{P_{d} g r^{-\alpha}}{I^{(o)}+\sigma^{2}}<T \mid r\right) .
\end{aligned}
$$

Do $g$ và $g^{\prime}$ là các biến ngẫu nhiên có hàm mật độ tuân theo hàm mũ nên ta có

$$
\begin{aligned}
& \mathbb{E}\left[\exp \left(-\hat{T} \frac{I^{(e)}+\sigma^{2}}{P_{d}^{(e)} r^{-\alpha}}\right)\left[1-\exp \left(T \frac{I^{(o)}+\sigma^{2}}{P_{d} r^{-\alpha}}\right)\right] \mid r\right] \\
& =\left[\begin{array}{l}
\exp \left(-\frac{T}{P_{d}} \frac{\sigma^{2}}{r^{-\alpha}}\right) \mathbb{E}\left[\exp \left(-\frac{\hat{T} I^{(e)}}{P_{d}^{(e)} r^{-\alpha}}\right) \mid r\right] \\
\quad-\exp \left(-\frac{\hat{I}}{P_{d}^{(e)}} \frac{\sigma^{2}}{r^{-\alpha}}\right)\left(1-\mathbb{E}\left[\exp \left(-\frac{T I^{(o)}}{P_{d} r^{-\alpha}}\right) \mid r\right]\right)
\end{array}\right] \\
& =\left[\begin{array}{c}
\exp \left(-\frac{T}{P_{d}} \frac{\sigma^{2}}{r^{-\alpha}}\right) \mathbb{E}\left[\exp \left(-\frac{\hat{I} I^{(e)}}{P_{d}^{(e)} r^{-\alpha}}\right) \mid r\right] \\
-\exp \left(-\left(\frac{T}{P_{d}}+\frac{\hat{T}}{P_{d}^{(e)}}\right) \frac{\sigma^{2}}{r^{-\alpha}}\right) \\
\quad \times \mathbb{E}\left[\exp \left(-\frac{\hat{T} I^{(e)}}{P_{d}^{(e)} r^{-\alpha}}\right) \exp \left(-\frac{T I^{(o)}}{P_{d} r^{-\alpha}}\right) \mid r\right]
\end{array}\right] .
\end{aligned}
$$

Xem xét biểu thức kỳ vọng thứ hai trong biểu thức (13), đặt là $\zeta(T, \hat{T}, \epsilon, r)$ và thay các giá trị $I^{(o)}$ và $I^{(s)}$ từ các phương trình (2) và (4), ta có

$$
\zeta(T, \hat{T}, \epsilon, r)=\mathbb{E}\left[\begin{array}{l}
\prod_{j \in \theta_{d}^{(e)}} \exp \left(-\frac{\hat{T} g_{j} l_{j}^{-\alpha}}{r^{-\alpha}}\right) \\
\times \prod_{j \in \theta_{u}^{(e)}} \exp \left(-\frac{\hat{T} P_{u}^{(s)} g_{u} d_{j}^{-\alpha}}{P_{d}^{(e)} r^{-\alpha}}\right) \\
\times \prod_{j \in \theta} \exp \left(-\frac{T g_{j} l_{j}^{-\alpha}}{r^{-\alpha}}\right)
\end{array}\right] .
$$

Ta lưu ý rằng $\theta$ chứa tập $\theta_{d}^{(e)}$ nhưng độc lập với $\theta_{u}^{(e)}$ nên $\zeta(T, \hat{T}, \epsilon, r)$ có thể được viết lại như sau:

$$
\mathbb{E}\left[\begin{array}{l}
\prod_{j \in \theta_{u}^{(e)}} \exp \left(-\frac{\hat{T} P_{u}^{(e)} d_{j}^{-\alpha}}{P_{d}^{(e)} r^{-\alpha}} g_{j}\right) \\
\times \prod_{j \in \theta \backslash \theta_{d}^{(e)}} \exp \left(-\frac{T l_{j}^{-\alpha}}{r^{-\alpha}} g_{j}\right) \\
\times \prod_{j \in \theta_{d}^{(e)}} \exp \left(-\frac{\hat{T} l_{j}^{-\alpha}}{r^{-\alpha}} g_{j}\right) \exp \left(-\frac{T l_{j}^{-\alpha}}{r^{-\alpha}} g_{j}\right)
\end{array}\right] .
$$

Do các giá trị độ lợi công suất của kênh là các biến Rayleigh độc lập nên ta có

$$
\zeta(T, \hat{T}, \epsilon, r)=\mathbb{E}\left[\begin{array}{l}
\prod_{j \in \theta_{u}^{(e)}} \frac{1}{1+\hat{T} \eta d_{j}^{-\alpha} r^{\alpha}} \\
\times \prod_{j \in \theta \backslash \theta_{d}^{(e)}} \frac{1}{1+T l_{j}^{-\alpha} r^{\alpha}} \\
\times \prod_{j \in \theta_{d}^{(e)}} \frac{1}{1+\hat{T} l_{j}^{-\alpha} r^{\alpha}} \frac{1}{1+T l_{j}^{-\alpha} r^{\alpha}},
\end{array}\right]
$$

trong đó $\eta=P_{u}^{(e)} / P_{d}^{(e)}$.

Sử dụng các tính chất của hàm sinh xác suất (PGF: Probability Generating Function) [13] với biến số là $d_{j}$ trong tập $\theta_{u}^{(e)}, l_{j}$ trong tập $\theta_{d}^{(e)}$, ta có

$$
\begin{aligned}
& \zeta(T, \hat{T}, \epsilon, r)= \\
& \exp \left(-2 \pi \lambda \frac{1-\epsilon}{\Delta} \int_{0}^{\infty}\left(1-\frac{1}{1+\hat{T} \eta d_{j}^{-\alpha} r^{\alpha}}\right) d_{j} d d_{j}\right) \\
& \times \exp \left(-2 \pi \lambda\left(1-\frac{\epsilon}{\Delta}\right) \int_{r}^{\infty}\left(1-\frac{1}{1+T l_{j}^{-\alpha} r^{\alpha}}\right) l_{j} l d_{j}\right) \\
& \times \exp \left(-2 \pi \lambda \frac{\epsilon}{\Delta} \int_{r}^{\infty}\left(1-\frac{1}{1+\hat{T} l_{j}^{-\alpha} r^{\alpha}} \frac{1}{1+T l_{j}^{-\alpha} r^{\alpha}}\right) l_{j} d l_{j}\right) .
\end{aligned}
$$

Sử dụng phương pháp đổi biến $y=\left(d_{j} / r\right)^{2}$ và $y=\left(l_{j} / r\right)^{2}$ đối với cả ba tích phân ở trên, ta thu được

$$
\begin{aligned}
& \exp \left(-2 \pi \lambda \frac{1-\epsilon}{\Delta} r^{2} \int_{0}^{\infty} \frac{\hat{T} \eta}{y^{\alpha / 2}+\hat{T} \eta} d y\right) \\
& \times \exp \left(-2 \pi \lambda\left(1-\frac{\epsilon}{\Delta}\right) r^{2} \int_{1}^{\infty}\left(1-\frac{1}{1+T y^{-\alpha / 2}}\right) d y\right) \\
& \times \exp \left(-2 \pi \lambda \frac{\epsilon}{\Delta} r^{2} \int_{1}^{\infty}\left(1-\frac{1}{1+\hat{T} y^{-\alpha / 2}} \frac{1}{1+T y^{-\alpha / 2}}\right) d y\right) .
\end{aligned}
$$

Sử dụng tính chất của hàm Gamma đối tích phân thứ nhất và đặt $\mathcal{L}(T, \hat{T})=\int_{1}^{\infty}\left(1-\frac{1}{1+\hat{T} y^{-\alpha / 2}} \frac{1}{1+T y^{-\alpha / 2}}\right) d y$, ta có

$$
\begin{aligned}
& \zeta(T, \hat{T}, \epsilon, r)= \\
& \exp \left(-\frac{2 \pi \lambda(1-\epsilon)}{\Delta \alpha}(\hat{T} \eta)^{2 / \alpha} \frac{\pi}{\sin \left(\frac{2 \pi}{\alpha}\right)} r^{2}\right) \\
& \quad \times \exp \left(-2 \pi \lambda\left(1-\frac{\epsilon}{\Delta}\right) \mathcal{L}(T, 0) r^{2}\right) \\
& \quad \times \exp \left(-2 \pi \lambda \frac{\epsilon}{\Delta} \mathcal{L}(T, \hat{T}) r^{2}\right) .
\end{aligned}
$$

Tương tự, giá trị kỳ vọng thứ 2 trong biểu thức (13) được tính dựa trên cách tính giá trị kỳ vọng thứ nhất với $T=0$ 
và mật độ trạm nhiễu là $\lambda / \Delta$. Cụ thể

$$
\begin{aligned}
\mathbb{E}\left[\exp \left(-\frac{\hat{T} I^{(e)}}{P_{d}^{(e)} r^{-\alpha}}\right) \mid r\right] \\
=\exp \left(-\frac{2 \pi \lambda(1-\epsilon)}{\alpha}(\hat{T} \eta)^{2 / \alpha} \sin \left(\frac{2 \pi}{\alpha}\right) r^{2}\right) \\
\quad \times \exp \left(-2 \pi \lambda \frac{\epsilon}{\Delta} \mathcal{L}(0, \hat{T}) r^{2}\right)
\end{aligned}
$$

\section{Tính $\mathbb{P}\left(\operatorname{SINR}^{(o)}<T \mid r\right)$}

Sử dụng các phép biến đổi tương tự như trên ta có

$$
\begin{aligned}
& \mathbb{P}\left(\operatorname{SINR}^{(o)}<T \mid r\right) \\
& \quad=1-\mathbb{P}\left(\frac{P_{d} g l^{-\alpha}}{\sum_{j \in \theta} P_{d} g_{j} l_{j}^{-\alpha}+\sigma^{2}}>T \mid r\right) \\
& \quad=1-\exp \left(-T \frac{\sigma^{2}}{P_{d} l^{-\alpha}}\right) \mathbb{E}\left[\prod_{j \in \theta} \exp \left(-T \frac{g_{j} l_{j}^{-\alpha}}{l^{-\alpha}} \mid r\right)\right] \\
& \quad=1-\exp \left(-T \frac{\sigma^{2}}{P_{d} l^{-\alpha}}\right) \exp \left(-2 \pi \lambda r^{2} \mathcal{L}(T, 0)\right) .
\end{aligned}
$$

Thay các biểu thức (17) và (18) vào biểu thức (11), đồng thời sử dụng phương pháp đổi biến $t=2 \pi \lambda r^{2}$, ta thu được xác suất phủ sóng của $\mathrm{CEU}$ như sau:

$\mathcal{P}(T, \hat{T}, \epsilon)=$

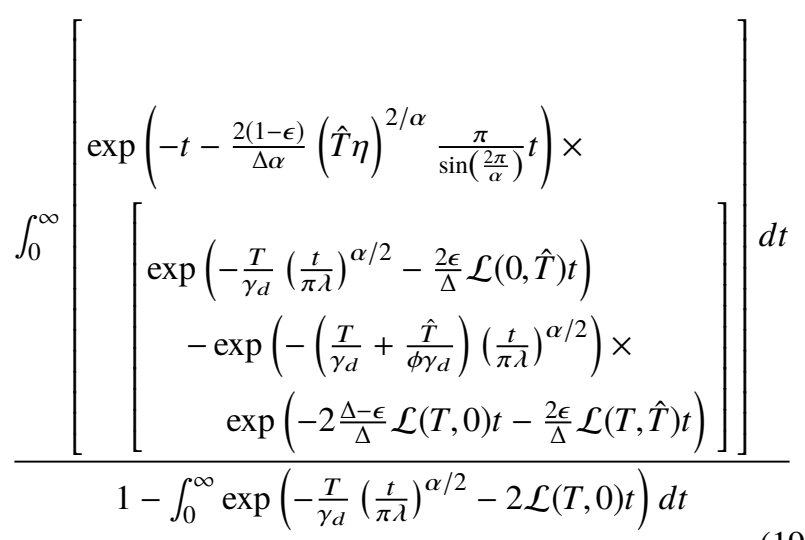

trong đó $\gamma_{d}=P_{d} / \sigma^{2}$ và $\gamma_{u}^{(e)}=P_{u}^{(e)} / \sigma^{2}$. Đồng thời $\eta=$ $\gamma_{u}^{(e)} / \gamma_{d}^{(e)}$.

Biểu thức (19) cho ta thấy được mối liên hệ xác suất phủ sóng của CEU với các tham số cấu hình hệ thống như tỉ lệ giữa số trạm phát cơ sở và trạm di động sử dụng cùng một sóng mang $\epsilon$, ngưỡng phân loại $\mathrm{CCU}$ và $\mathrm{CEU} T$ và độ nhạy của thiết bị $\hat{T}$. Kết quả này là đóng góp chính của bài báo này.

\section{Các trường hợp đặc biệt}

a) Không sủ dụng TDD linh động: Trong trường hợp này, không có sự chia sẻ về sóng mang trạm phát cơ sở và trạm di đông. Tức là $\epsilon=1$. Khi đó xác suất phủ sóng của CEU là

$$
\begin{aligned}
& \mathcal{P}(T, \hat{T}, \epsilon)= \\
& \int_{0}^{\infty}\left[\begin{array}{c}
\exp \left(-t-\frac{T}{\gamma_{d}}\left(\frac{t}{\pi \lambda}\right)^{\alpha / 2}-\frac{2}{\Delta} \mathcal{L}(0, \hat{T}) t\right) \\
-\exp \left(-\left(\frac{T}{\gamma_{d}}+\frac{\hat{T}}{\phi \gamma_{d}}\right)\left(\frac{t}{\pi \lambda}\right)^{\alpha / 2}\right) \\
\times \exp \left(-2 \frac{\Delta-1}{\Delta} \mathcal{L}(T, 0) t-\frac{2 \epsilon}{\Delta} \mathcal{L}(T, \hat{T}) t\right)
\end{array}\right] d t \\
& 1-\int_{0}^{\infty} \exp \left(-\frac{T}{\gamma_{d}}\left(\frac{t}{\pi \lambda}\right)^{\alpha / 2}-2 \mathcal{L}(T, 0) t\right) d t
\end{aligned}
$$

Kết quả này cho thấy xác suất phủ sóng của người dùng khi không sử dụng kỹ thuật TDD linh động. Kết quả này đã được trình bày trong các Tài liệu trước đây như [14].

b) $P_{u}$ và $P_{d}$ vô cùng lớn so với $\sigma^{2}$ : Khi đó $\gamma_{d} \rightarrow \infty$ và $\gamma_{u} \rightarrow \infty$. Xác suất phủ sóng của $\mathrm{CEU}$ trong trường hợp này là

$$
\begin{aligned}
& \mathcal{P}(T, \hat{T}, \epsilon)= \\
& \int_{0}^{\infty}\left[\begin{array}{c}
\exp \left(-t-\frac{2(1-\epsilon)}{\Delta \alpha}(\hat{T} \eta)^{2 / \alpha} \frac{\pi}{\sin \left(\frac{2 \pi}{\alpha}\right)} t\right) \\
\times\left[\begin{array}{c}
\exp \left(-\frac{2 \epsilon}{\Delta} \mathcal{L}(0, \hat{T}) t\right) \\
-\exp \left(-2 \frac{\Delta-\epsilon}{\Delta} \mathcal{L}(T, 0) t-\frac{2 \epsilon}{\Delta} \mathcal{L}(T, \hat{T}) t\right)
\end{array}\right]
\end{array}\right] d t \\
& \frac{\left[\begin{array}{c}
1-\int_{0}^{\infty} \exp (-2 \mathcal{L}(T, 0) t) d t \\
1+\frac{2(1-\epsilon)}{\Delta \alpha}(\hat{T} \eta)^{2 / \alpha} \frac{\pi}{\sin \left(\frac{2 \pi}{\alpha}\right)}+\frac{2 \epsilon}{\Delta} \mathcal{L}(0, \hat{T})
\end{array}\right]}{\left.\frac{1}{1+\frac{2(1-\epsilon)}{\Delta \alpha}(\hat{T} \eta)^{2 / \alpha} \frac{\pi}{\sin \left(\frac{2 \pi}{\alpha}\right)}+2 \frac{\Delta-\epsilon}{\Delta} \mathcal{L}(T, 0)+\frac{2 \epsilon}{\Delta} \mathcal{L}(T, \hat{T})}\right]} \\
& \left.=\frac{1-\frac{1}{1+2 \mathcal{L}(T, 0)}}{1}\right]
\end{aligned}
$$

Thông thường đối với một hệ thống mạng thông tin di động, công suất của các tram phát thường rất lớn so với công suất ồn Gauss. Do đó, biểu thức (20) có thể dùng để đánh giá hiệu suất của kỹ thuật TDD linh động và kỹ thuật tái sử dụng tần số ở lớp vật lý.

\section{MÔ PHỎNG VÀ ĐÁNH GIÁ}

Trong phần này, chúng ta sẽ sử dụng phần mềm Matlab để kiểm nghiệm tính đúng đắn của kết quả phân tích lý thuyết thông qua mô phỏng Monte Carlo. Đồng thời chúng ta đưa ra các nhận xét về ảnh hưởng của các tham số mạng tới xác suất phủ sóng của CEU.

Theo khuyến nghị của $3 \mathrm{GPP}$, công suất tối đa của một trạm di động và tối thiểu một trạm cơ sở trong mạng LTE lần lượt $33 \mathrm{dBm}$ và $53 \mathrm{dBm}$ [15]. Do đó trong phần này ta sử dụng tỉ số $\eta=0,01$. Bên cạnh đó, giá trị của $\phi$ thay đổi 


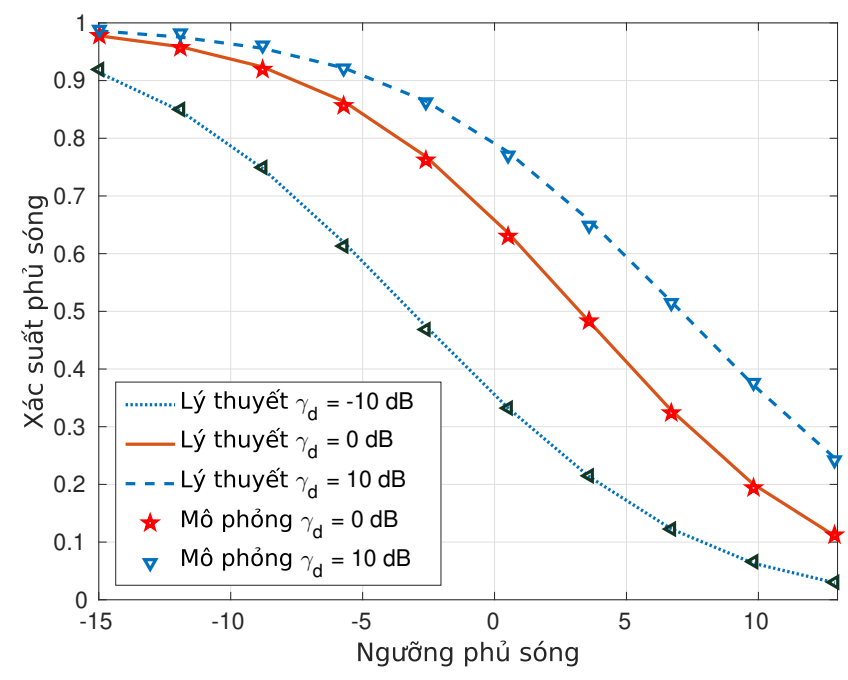

Hình 4. So sánh kết quả lý thuyết và mô phỏng.

tùy vào tính chất của mạng, nhưng các khuyến nghị đưa ra xác định $\phi$ dao động từ 2 đến 20 [16]. Do đó, trong bài báo này ta sử dụng $\phi=10$. Dựa theo kết quả từ hình 4 , chúng ta thấy các đường liền thể hệ kết quả lý thuyết trùng khớp với các đường tạo bởi các điểm rời rạc thể hiện giá trị mô phỏng. Do đó chúng ta có thể kết luận về sự hợp lý của phương pháp phân tích lý thuyết.

Theo hình 4 ta thấy khi ngưỡng phủ sóng $\hat{T}$ tăng, xác suất phủ sóng của người dùng giảm mạnh. Ví dụ đối với trường hợp tỉ số $\gamma_{d}=0 \mathrm{~dB}$, khi ngưỡng phủ sóng tăng từ $\hat{T}=-2,6 \mathrm{~dB}$ lên đến $\hat{T}=3,6$ thì xác suất phủ sóng giảm $37,22 \%$ từ 0,7684 xuống 0,4824 . Điều này hoàn toàn hợp lý với hệ thống ngoài thực tế vì $\hat{T}$ đại diện cho yêu cầu về đổ lớn tỉ số SINR của thiết bị thu để có thể thực hiện truyền tin. Khi $\hat{T}$ tăng đồng nghĩa với việc độ nhạy của thiết bị kém đi và nó cần một tỉ số SINR lớn hơn để truyền tin. Đồng thời, tỉ số SINR lại không phụ thuộc vào $\hat{T}$. Do đó, khi $\hat{T}$ tăng thì xác suất phủ sóng của người dùng giảm.

Cũng theo hình 4 , ta thấy xác suất phủ sóng tăng khi ta tăng tỉ số công suất phát của trạm phát với công suất ồn Gauss $\gamma_{d}$. Ví dụ với trường hợp $\hat{T}=0,5 \mathrm{~dB}$, khi tỉ số $\gamma_{d}=0 \mathrm{~dB}$ tăng lên $\gamma_{d}=10 \mathrm{~dB}$ thì xác suất phủ sóng tăng $21,06 \%$ từ 0,6367 lên 0,7708 . Ta lưu ý rằng khi ta tăng tỉ số $\gamma_{d}$ có nghĩa là ta tăng công suất phát của tất cả các trạm phát, bao gồm cả trạm nhiễu và trạm phục vụ. Do đó trong trường hợp này, ta có thể kết luận rằng: việc tăng công suất phát có thể được sử dụng để tăng xác suất phủ sóng của người dùng. Các kết luận tương tự với mô hình không sử dụng TDD linh động cũng đã được đưa trong các kết quả nghiên cứu liên quan.

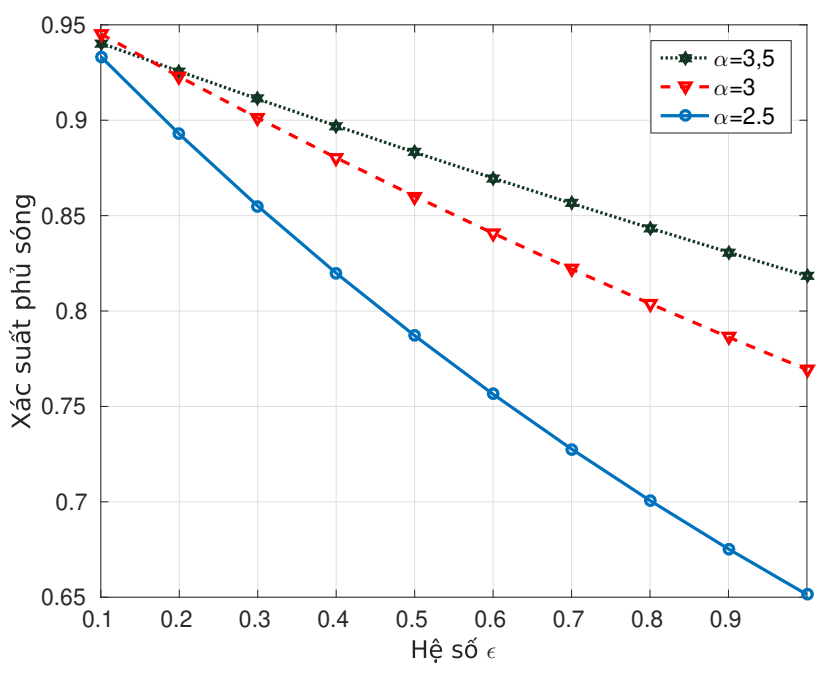

Hình 5. Ảnh hưởng của hệ số $\epsilon$ lên xác suất phủ sóng.

\section{1. Ảnh hưởng của hệ số $\epsilon$ lên xác suất phủ sóng}

Hình 5 cho ta thấy được sự ảnh hưởng của hệ số $\epsilon$ lên xác suất phủ sóng của người dùng. Ta nhắc lại rằng, một người dùng trong mạng luôn chịu nhiễu từ cùng số lượng trạm phát (trạm di động, trạm cơ sở) khi tỉ số giữa trạm cơ sở với trạm di động sử dụng cùng một sóng mang tại cùng một thời điểm $\epsilon$ thay đổi. Khi ta giảm $\epsilon$, số lượng trạm nhiễu là trạm cơ sở giảm và số trạm nhiễu là trạm di động tăng. Với trường hợp đặc biệt $\epsilon=1$ tương ứng với trường hợp không sử dụng TDD linh động, tức là không có sự chia sẻ sóng mang giữa trạm cơ sở và trạm di động. Khi đó, trạm di động chỉ chịu nhiễu từ các trạm cơ sở. Đối với trường hợp $\epsilon=0$ hay $1-\epsilon=1$ tức là tất cả trạm nhiễu của người dùng ta đang xét là trạm di động. Do công suất phát của các trạm di động luôn luôn nhỏ hơn công suất phát của các trạm cơ sở nên khi $\epsilon$ giảm, tổng công suất nhiễu của người dùng giảm. Điều này kéo theo tỉ số SINR và xác suất phủ sóng tăng.

Ví dụ đối với trường hợp hệ số $\epsilon$ giảm từ 0,8 xuống 0,4 tương đương với số trạm nhiễu là trạm cơ sở giảm từ $80 \%$ xuống $40 \%$, xác suất phủ sóng của người dùng tăng lên $9 \%$ từ đến 0,8083 lên 0,8803 .

\section{2. Ảnh hưởng của hệ số $\lambda$ lên xác suất phủ sóng}

Hình 6 cho ta thấy được ảnh hưởng của mật độ trạm phát lên xác suất phủ sóng của người dùng. Ta thây khi ta tăng mật độ trạm phát thì xác suất phủ sóng tăng nhưng nhanh chóng đạt đến ngưỡng và không thay đổi. Kết luận này cũng tương tự như các kết luận đã được tìm ra trong các nghiên cứu trước đây như trong [17].

Hình 6 chỉ ra rằng người dùng đạt được cùng một giá trị xác suất phủ sóng là 9,04 tại hai bộ số $(\lambda=0,1$ và $\epsilon=0,2)$ 


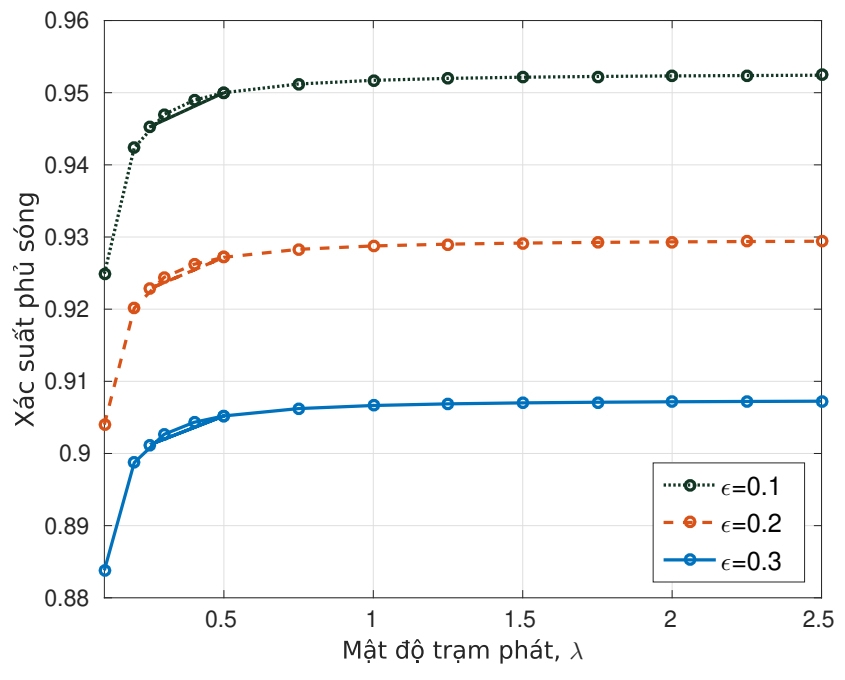

Hình 6. Ảnh hưởng của hệ số $\lambda$ lên xác suất phủ sóng.

và $(\lambda=0,4$ và $\lambda=0,3)$. Khi $\lambda$ giảm từ 0,4 xuống 0,1 thì mật độ trạm giảm đi $75 \%$, trong khi đó khi $\epsilon$ giảm từ 0,3 xuống 0,2 thì mật độ trạm cơ sơ phát trên cùng một sóng mang tại cùng một thời điểm chỉ giảm đi 33,3\%. Do đó, ta có thể nói rằng để nâng cao xác suất phủ sóng việc giảm $\epsilon$ có hiệu quả hơn việc tăng $\lambda$.

\section{KẾT LUẬN}

Bài báo đã sử dụng mô hình xác suất thống kê để mô hình hóa đường xuống của mạng thông tin di động LTE sử dụng đồng thời kỹ thuật tái sử dụng tần số cứng và kỹ thuật TDD linh động. Trong đó xác suất phủ sóng của người cùng biên $\mathrm{CEU}$ được tập trung đánh giá. Bài báo đã đưa ra biểu tính tính xác suất phủ sóng của CEU trong môi trường pha-đinh Rayleigh. Các kết quả mô phỏng và phân tích đã chỉ ra rằng: (i) kỹ thuật TDD động có thể cái thiện một các rõ ràng xác suất phủ sóng của $\mathrm{CEU;} \mathrm{(ii)} \mathrm{để}$ tăng xác suất phủ sóng của CEU thì việc giảm số trạm cơ sở phát trên cùng một sóng mang mang lại hiệu quả hơn việc tăng số lượng trạm cơ sở trong mạng. Tuy nhiên, để đánh giá chính xác hiệu suất của kỹ thuật TDD linh động, xác suất phủ sóng của người dùng trên đường lên cũng cần được xem xét và tính toán cụ thể.

\section{TÀI LIỆU THAM KHẢO}

[1] D. J. Daley and D. Vere-Jones, 5G NR: Architecture, Technology, Implementation, and Operation of 3GPP New Radio Standards. Academic Press, 2019.

[2] 3GPP, "3GPP TS 38.104 version 15.2.0 release 15: 5G; NR; Base station (BS) radio transmission and reception," 2018.

[3] F. Khan, LTE for 4G Mobile Broadband: Air Interface Technologies and Performance. Cambridge University Press, 2009

[4] C. Christopher, An Introduction to LTE: LTE, LTEAdvanced, SAE, VoLTE and $4 G$ Mobile Communications. Wiley, 2014.
[5] A. S. Hamza, S. S. Khalifa, H. S. Hamza, and K. Elsayed, "A survey on inter-cell interference coordination techniques in OFDMA-based cellular networks," IEEE Commun. Surveys \& Tutorials, vol. 15, no. 4, pp. 1642-1670, 2013.

[6] M. Kamel, W. Hamouda, and A. Youssef, "Ultra-dense networks: A survey," IEEE Communications Surveys Tutorials, vol. 18, no. 4, pp. 2522-2545, 2016.

[7] M. Ding, D. Lopez-Porez, R. Xue, A. V. Vasilakos, and W. Chen, "On dynamic time-division-duplex transmissions for small-cell networks," IEEE Transactions on Vehicular Technology, vol. 65, no. 11, pp. 8933-8951, Nov 2016.

[8] Y. Ramamoorthi and A. Kumar, "Dynamic time division duplexing for downlink/uplink decoupled millimeter wave based cellular networks," IEEE Communications Letters, pp. $1-1,2019$.

[9] M. N. Kulkarni, J. G. Andrews, and A. Ghosh, "Performance of dynamic and static TDD in self-backhauled millimeter wave cellular networks," IEEE Transactions on Wireless Communications, vol. 16, no. 10, pp. 6460-6478, Oct 2017.

[10] T. Ding, M. Ding, G. Mao, Z. Lin, A. Y. Zomaya, and D. Lopez-Perez, "Performance analysis of dense small cell networks with dynamic TDD," IEEE Transactions on Vehicular Technology, vol. 67, no. 10, pp. 9816-9830, Oct 2018.

[11] B. Yu, L. Yang, H. Ishii, and S. Mukherjee, "Dynamic TDD support in macrocell-assisted small cell architecture," IEEE Journal on Selected Areas in Communications, vol. 33, no. 6, pp. 1201-1213, June 2015.

[12] A. K. Gupta, M. N. Kulkarni, E. Visotsky, F. W. Vook, A. Ghosh, J. G. Andrews, and R. W. Heath, "Rate analysis and feasibility of dynamic TDD in 5G cellular systems," in 2016 IEEE International Conference on Communications (ICC), May 2016, pp. 1-6.

[13] M. A. Stegun and I. A., Handbook of Mathematical Functions with Formulas, Graphs, and Mathematical Tables, 9th ed. Dover Publications, 1972.

[14] S. C. Lam, "Performance analysis of fractional frequency reuse in random cellular networks," $\mathrm{PhD}$ dissertation, University of Technology Sydney, 2018.

[15] 3GPP TR 36.828 V11.0, "E-UTRA further enhancements to LTE time division duplex (TDD) for downlink-uplink (DLUL) interference management and traffic adaptation," June 2012.

[16] 3GPP-TSG-RAN-R1-050738. Interference mitigation? Considerations and results on frequency reuse. September, 2005, Siemens.

[17] J. G. Andrews, F. Baccelli, and R. K. Ganti, "A new tractable model for cellular coverage," in 2010 48th Annu. Allerton Conference on Communication, Control, and Computing (Allerton), pp. 1204-1211.

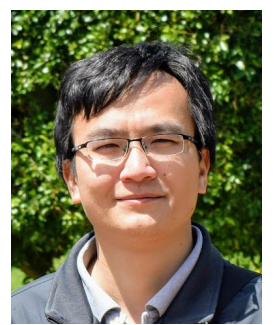

Lâm Sinh Công tốt nghiệp Đại học ngành Điện tử - Viễn thông năm 2010 và Thạc sĩ ngành Kỹ thuật Điện tử năm 2012 tại Trường Đại học Công nghệ, Đại học Quốc gia Hà Nội, tốt nghiệp Tiến sĩ tại trường Đại học Công nghệ Sydney, Úc năm 2018. Hiện nay, tác giả là giảng viên Trường Đại học Công nghệ, Đại học Quốc gia Hà Nội. Hướng nghiên cứu chính của tác giả là mô hình hóa, mô phỏng và đánh giá hệ thông tin di động thế hệ mới $(4 \mathrm{G}, 5 \mathrm{G})$. 